\title{
PRECONDITIONED GLOBAL KRYLOV SUBSPACE METHODS FOR SOLVING SADDLE POINT PROBLEMS WITH MULTIPLE RIGHT-HAND SIDES*
}

\author{
A. BADAHMANE ${ }^{\dagger \ddagger}$, A. H. BENTBIB ${ }^{\dagger}$, AND H. SADOK ${ }^{\ddagger}$
}

\begin{abstract}
In the present paper, we propose a preconditioned global approach as a new strategy to solve linear systems with several right-hand sides coming from saddle point problems. The preconditioner is obtained by replacing a $(2,2)$-block in the original saddle-point matrix $\mathcal{A}$ by another well-chosen block. We apply the global GMRES method to solve this new problem with several right-hand sides and give some convergence results. Moreover, we analyze the eigenvalue distribution and the eigenvectors of the proposed preconditioner when the first block is positive definite. We also compare different preconditioned global Krylov subspace algorithms (CG, MINRES, FGMRES, GMRES) with preconditioned block (CG, GMRES) algorithms. Numerical results show that our preconditioned global GMRES method is competitive with other preconditioned global Krylov subspace and preconditioned block Krylov subspace methods for solving saddle point problems with several right-hand sides.
\end{abstract}

Key words. global Krylov subspace method, GMRES, MINRES, CG, preconditioner, saddle point problem

AMS subject classifications. 65F10, 65N22, 65F50

1. Introduction. Many problems in science and engineering require the solution of saddle point problems with multiple right-hand sides (see, for example $[5,8]$ ),

$$
\underbrace{\left[\begin{array}{cc}
A & B^{T} \\
\epsilon B & O
\end{array}\right]}_{\mathcal{A}} \underbrace{\left[\begin{array}{c}
X \\
Y
\end{array}\right]}_{\mathcal{X}}=\underbrace{\left[\begin{array}{c}
F \\
\epsilon G
\end{array}\right]}_{\mathcal{B}},
$$

where $A \in \mathbb{R}^{n \times n}$ is a symmetric matrix and $B^{T} \in \mathbb{R}^{n \times m}$ has full column rank with $X \in \mathbb{R}^{n \times s}, Y \in \mathbb{R}^{m \times s}, F \in \mathbb{R}^{n \times s}, G \in \mathbb{R}^{m \times s}$, and $\epsilon= \pm 1$. Here $B^{T}$ denotes the transpose of $B$. Since the matrices $A$ and $B$ in (1.1) are large and sparse, the solution of (1.1) with $s=1$ is suited for the solution by an iterative method. Many effective iterative methods have been developed for solving saddle point problems such as the Hermitian and skew-Hermitian splitting (HSS) method [3] and the Regularized HSS iteration method [2].

Instead of applying a standard iterative method to the solution of each one of the saddle point problems

$$
\mathcal{A} \mathcal{X}^{(i)}=b^{(i)}, \quad \text { for } i=1, \ldots, s,
$$

independently, it is often more efficient to apply a global method to (1.1). We use the global version of GMRES for the solution of nonsymmetric saddle point problems with multiple right-hand sides (1.1). This version of GMRES has been introduced in [13] and studied in [4]. The method is based on the global version of the standard Arnoldi process; see for example [1]. However, Krylov subspace methods without a good preconditioner converge very slowly when applied to saddle point problems with multiple right-hand sides. In order to accelerate the convergence, several preconditioners and iterative methods have been proposed for the solution of (1.1) with a single right-hand side vector $[2,7,6,12]$.

\footnotetext{
* Received October 28, 2018. Accepted November 11, 2019. Published online on December 12, 2019. Recommended by L. Reichel.

${ }^{\dagger}$ Laboratoire de Mathématiques Appliquées et Informatique, Faculté des Sciences et Techniques Gueliz, Marrakech, Morocco (a.bentbib@uca.ac.ma).

‡Laboratoire de Mathématiques Pures et Appliquées, Université du Littoral Côte d’Opale, Batiment H. Poincarré, 50 rue F. Buisson, F-62280 Calais Cedex, France (badahmane.achraf@gmail.com, sadok@univ-littoral.fr).
} 
In this work, we present variants of preconditioned global Krylov subspace methods that yield better numerical results compared to the block approach used in $[5,8]$ for solving (1.1). The paper is organized as follows. An example of modeling that leads to this type of system is outlined in Section 2. In Section 3 we give the first-order optimality condition for a block quadratic problem. In Section 4 we review some properties and definitions of global Krylov subspace methods. In Section 5 we introduce the preconditioner $\mathcal{P}_{\epsilon, \alpha, Q}$, and we also analyze the eigenvalue distribution and the eigenvectors of the proposed preconditioner. Numerical experiments are discussed in Section 6. We use the following notation: For two matrices $Y$ and $Z$ in $\mathbb{R}^{(n+m) \times s}$, we define the inner product $\langle Y, Z\rangle_{F}=\operatorname{trace}\left(Y^{T} Z\right)$. The associated norm is the Frobenius norm denoted by $\|\cdot\|_{F}$. A system of vectors (matrices) of $\mathbb{R}^{(n+m) \times s}$ is said to be F-orthonormal if it is orthonormal with respect to the scalar product $\langle., .\rangle_{F}$. Finally, the Kronecker product of the matrices $C$ and $D$ is given by $C \otimes D=\left[c_{i, j} D\right]$.

2. The problem. Saddle point problems with multiple right-hand sides appear when we apply the feedback control approach [5] to the following non-stationary Stokes equations:

$$
\left\{\begin{aligned}
\frac{\partial u(t, x)}{\partial t}-v \Delta u(t, x)+\nabla p(t, x) & =0, & & (0, \infty) \times \Omega \\
\nabla \cdot u(t, x) & =0, & & (0, \infty) \times \Omega .
\end{aligned}\right.
$$

Here $t \in(0, \infty), x \in \Omega, v>0$ is the viscosity, and $u$ and $p$ denote the velocity and the pressure, respectively. Moreover, $\Omega \subset \mathbb{R}^{2}$ is a domain with boundary $\partial \Omega$. Some Dirichlet boundary conditions which describe an inflow-outflow problem with adequate initial conditions are found in [11]. By using the feedback control approach [5], we obtain a saddle point problem with multiple right-hand sides.

3. The classification of the solution of the saddle point problem with multiple righthand sides (1.1). We assume that $A$ is a positive definite matrix. With the use of the Frobenius inner product $\langle., .\rangle_{F}$, equation (1.1) arises from a quadratic constrained optimization problem

$$
\left\{\begin{aligned}
\min _{X} J(X) & =\min _{X} \frac{1}{2}\langle A X, X\rangle_{F}-\langle X, F\rangle_{F} \\
B X & =G
\end{aligned}\right.
$$

We define the Lagrangian $\mathcal{L}$ as

$$
\mathcal{L}(X, Y)=\frac{1}{2}\langle A X, X\rangle_{F}-\langle X, F\rangle_{F}+\langle Y, B X-G\rangle_{F}
$$

THEOREM 3.1. The solution of (1.1) is a saddle point of the Lagrangian (3.1).

Proof. To show that the solution of (1.1) is a saddle point of (3.1), we start discussing the differentiability of $\mathcal{L}$ and calculate its differential. As we know, the function

$$
\begin{aligned}
\mathbb{R}^{n \times s} \times \mathbb{R}^{n \times s} & \rightarrow \mathbb{R} \\
(X, Y) & \rightarrow \operatorname{Trace}\left(Y^{T} X\right)
\end{aligned}
$$


is differentiable. Therefore, the functional $\mathcal{L}$ is differentiable. Next we calculate the differential of $\mathcal{L}$. Let $H \in \mathbb{R}^{n \times s}$. We have

$$
\begin{aligned}
\mathcal{L}(X+H, Y)= & \frac{1}{2}\langle A(X+H), X+H\rangle_{F}-\langle X+H, F\rangle_{F}+\langle Y, B(X+H)-G\rangle_{F} \\
= & \frac{1}{2}\langle A X, X\rangle_{F}+\langle A H, X\rangle_{F}+\frac{1}{2}\langle A H, H\rangle_{F}-\langle X, F\rangle_{F} \\
& \quad-\langle H, F\rangle_{F}+\langle Y, B X-G\rangle_{F}+\langle Y, B H\rangle_{F} \\
= & \frac{1}{2}\langle A X, X\rangle_{F}-\langle X, F\rangle_{F}+\langle Y, B X-G\rangle_{F}+\langle A H, X\rangle_{F} \\
& \quad+\frac{1}{2}\langle A H, H\rangle_{F}-\langle H, F\rangle_{F}+\langle Y, B H\rangle_{F} \\
= & \mathcal{L}(X, Y)+\langle A H, X\rangle_{F}+\frac{1}{2}\langle A H, H\rangle_{F}-\langle H, F\rangle_{F}+\langle Y, B H\rangle_{F} .
\end{aligned}
$$

This can be written as

$$
\begin{aligned}
\mathcal{L}(X+H, Y)-\mathcal{L}(X, Y)-\frac{1}{2}\langle A H, H\rangle_{F} & =\langle A X, H\rangle_{F}-\langle H, F\rangle_{F}+\langle Y, B H\rangle_{F} \\
& =\left\langle A X-F+B^{T} Y, H\right\rangle_{F} .
\end{aligned}
$$

Therefore

$$
d_{X} \mathcal{L}(X, Y)=A X-F+B^{T} Y
$$

Similarly, we obtain

$$
d_{Y} \mathcal{L}(X, Y)=B X-G .
$$

According to (3.2) and (3.3), we conclude that the solution $\left(X_{*}, Y_{*}\right)$ of (1.1) is a critical point of the functional $\mathcal{L}$. To complete the proof, it must be shown that $\left(X_{*}, Y_{*}\right)$ satisfies

$$
\mathcal{L}\left(X_{*}, Y\right) \leq \mathcal{L}\left(X_{*}, Y_{*}\right) \leq \mathcal{L}\left(X, Y_{*}\right) \quad \text { for any } X \in \mathbb{R}^{n \times s} \text { and } Y \in \mathbb{R}^{m \times s} .
$$

We have

$$
\begin{aligned}
\mathcal{L}(X, Y) & =\frac{1}{2} \operatorname{Trace}\left(\left[\begin{array}{c}
X \\
Y
\end{array}\right]^{T}\left[\begin{array}{cc}
A & B^{T} \\
\epsilon B & O
\end{array}\right]\left[\begin{array}{l}
X \\
Y
\end{array}\right]\right)-\operatorname{Trace}\left(\left[\begin{array}{c}
X \\
Y
\end{array}\right]^{T}\left[\begin{array}{c}
F \\
\epsilon G
\end{array}\right]\right) \\
& =\frac{1}{2} \operatorname{Trace}\left(\left[\begin{array}{c}
X \\
Y
\end{array}\right]^{T}\left[\begin{array}{cc}
A & B^{T} \\
\epsilon B & O
\end{array}\right]\left[\begin{array}{l}
X \\
Y
\end{array}\right]\right)-\operatorname{Trace}\left(\left[\begin{array}{c}
X \\
Y
\end{array}\right]^{T}\left[\begin{array}{cc}
A & B^{T} \\
\epsilon B & O
\end{array}\right]\left[\begin{array}{c}
X_{*} \\
Y_{*}
\end{array}\right]\right) .
\end{aligned}
$$

Then

$$
\mathcal{L}\left(X_{*}, Y_{*}\right)=-\frac{1}{2} \operatorname{Trace}\left(\left[\begin{array}{c}
X_{*} \\
Y_{*}
\end{array}\right]^{T}\left[\begin{array}{cc}
A & B^{T} \\
\epsilon B & O
\end{array}\right]\left[\begin{array}{c}
X_{*} \\
Y_{*}
\end{array}\right]\right)
$$

Consequently,

$$
\mathcal{L}(X, Y)-\mathcal{L}\left(X_{*}, Y_{*}\right)=\frac{1}{2} \operatorname{Trace}\left(\left[\begin{array}{c}
X-X_{*} \\
Y-Y_{*}
\end{array}\right]^{T}\left[\begin{array}{cc}
A & B^{T} \\
\epsilon B & O
\end{array}\right]\left[\begin{array}{c}
X-X_{*} \\
Y-Y_{*}
\end{array}\right]\right) .
$$

Case 1: $X=X_{*}$ : Then

$$
\mathcal{L}\left(X_{*}, Y\right)-\mathcal{L}\left(X_{*}, Y_{*}\right)=0
$$

Case 2: $Y=Y_{*}$ : Then

$$
\mathcal{L}\left(X, Y_{*}\right)-\mathcal{L}\left(X_{*}, Y_{*}\right)=\operatorname{Trace}\left(\left(X-X_{*}\right)^{T} A\left(X-X_{*}\right)\right) \geq 0 .
$$

We conclude that $\left(X_{*}, Y_{*}\right)$ is a saddle point of the Lagrangian $\mathcal{L}$. 
4. Global Krylov subspace methods. In this section we recall some properties and definitions of the global methods [9, 14].

DEFINITION 4.1. The global Krylov subspace $\mathcal{K}_{k}(\mathcal{A}, V)$ is the subspace spanned by the matrices $V, \mathcal{A} V, \ldots, \mathcal{A}^{k-1} V$.

REMARK 4.2. Let $V$ be a real matrix of dimension $(n+m) \times s$. According to the definition of the subspace $\mathcal{K}_{k}(\mathcal{A}, V)$, we have

$$
\mathcal{Z} \in \mathcal{K}_{k}(\mathcal{A}, V) \Longleftrightarrow \mathcal{Z}=\sum_{i=1}^{k} \alpha_{i} \mathcal{A}^{i-1} V, \quad \alpha_{i} \in \mathbb{R}, i=1, \ldots, k .
$$

In other words, $\mathcal{K}_{k}(\mathcal{A}, V)$ is the subspace of $\mathbb{R}^{(n+m) \times s}$ that contains all the matrices of dimension $(n+m) \times s$, written as $\mathcal{Z}=P(\mathcal{A}) V$, where $P$ is a polynomial of degree at most $k-1$.

DEFinition 4.3 (Diamond Product). Let $Y=\left[Y_{1}, Y_{2}, \ldots, Y_{p}\right]$ and $Z=\left[Z_{1}, Z_{2}, \ldots, Z_{l}\right]$ be matrices of dimensions $n \times p s$ and $n \times l s$, respectively, where $Y_{i}$ and $Z_{j}(i=1, \ldots, p$, $j=1, \ldots, l)$ are $n \times s$ matrices. The product $\diamond$ is defined by

$$
Y^{T} \diamond Z=\left[\begin{array}{cccc}
\left\langle Y_{1}, Z_{1}\right\rangle_{F} & \left\langle Y_{1}, Z_{2}\right\rangle_{F} & \ldots & \left\langle Y_{1}, Z_{l}\right\rangle_{F} \\
\left\langle Y_{2}, Z_{1}\right\rangle_{F} & \left\langle Y_{2}, Z_{2}\right\rangle_{F} & \ldots & \left\langle Y_{2}, Z_{l}\right\rangle_{F} \\
\vdots & \vdots & \ddots & \vdots \\
\left\langle Y_{p}, Z_{1}\right\rangle_{F} & \left\langle Y_{p}, Z_{2}\right\rangle_{F} & \ldots & \left\langle Y_{p}, Z_{l}\right\rangle_{F}
\end{array}\right] \in \mathbb{R}^{p \times l} .
$$

We use the global Arnoldi process for building an F-orthonormal basis of $\mathcal{K}_{k}(\mathcal{A}, V)$. Let $X_{0}$ be the initial approximate solution of (1.1), and let $R_{0}=\mathcal{B}-\mathcal{A} X_{0}$ be the corresponding residual. The following global Arnoldi process, Algorithm 1, which is based on the modified Gram-Schmidt process, constructs an F-orthonormal basis $V_{1}, V_{2}, \ldots, V_{k}$ of $\mathcal{K}_{k}\left(\mathcal{A}, R_{0}\right)$. In other words

$$
\operatorname{Trace}\left(V_{i}^{T} V_{j}\right)=\delta_{i j}, \quad \text { for } i, j=1, \ldots, k,
$$

where $\delta_{i j}$ is the Kronecker symbol with $\delta_{i j}=0$ for $i \neq j$ and $\delta_{i i}=1$.

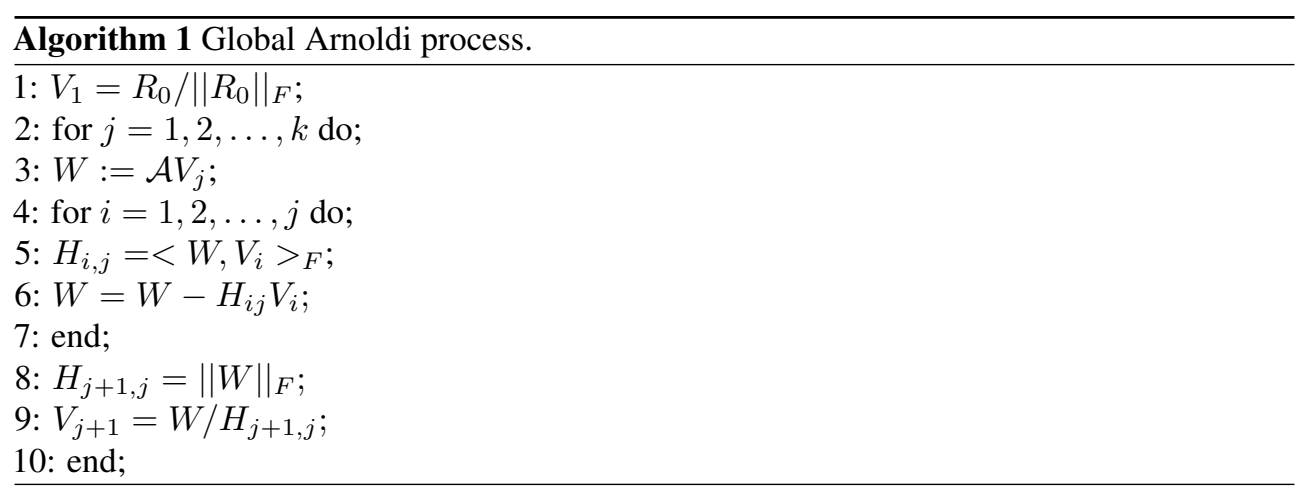

Proposition 4.4. Assume that $h_{i+1, i} \neq 0$, for $i=1, \ldots, k$, and let $\mathcal{V}_{k}$ be the matrix defined by $\mathcal{V}_{k}=\left[V_{1}, \ldots, V_{k}\right]$, where $V_{i}$, for $i=1, \ldots, k$, are the matrices computed by Algorithm 1. We have the following relations:

$$
\begin{aligned}
& \mathcal{A} \mathcal{V}_{k}=\mathcal{V}_{k} \diamond H_{k}+H_{k+1, k}\left[O, \ldots, O, V_{k+1}\right], \\
& \mathcal{A} \mathcal{V}_{k}=\tilde{\mathcal{V}}_{k} \diamond \tilde{H}_{k},
\end{aligned}
$$


where $\tilde{\mathcal{V}}_{k}=\left[V_{1}, \ldots, V_{k}, V_{k+1}\right] \in \mathbb{R}^{(n+m) \times(k+1) s}$ and $\tilde{H}_{k} \in \mathbb{R}^{(k+1) \times k}$. The global GMRES method constructs, at step $k$, the approximation $X_{k}$ satisfying the following two relations

$$
X_{k}-X_{0} \in \mathcal{K}_{k}\left(\mathcal{A}, R_{0}\right) \quad \text { and } \quad\left\langle\mathcal{A}^{j} R_{0}, R_{k}\right\rangle_{F}=0, \quad \text { for } j=1, \ldots, k .
$$

The residual $R_{k}=\mathcal{B}-\mathcal{A} X_{k}$ satisfies the minimization property

$$
\left\|R_{k}\right\|_{F}=\min _{Z \in \mathcal{K}_{k}\left(\mathcal{A}, R_{0}\right)}\left\|R_{0}-\mathcal{A} Z\right\|_{F} .
$$

Problem (4.1) is solved by applying the global Arnoldi process.

4.1. Convergence analysis of the global GMRES method. In this section, we recall a bound for the residual $R_{k}$ in (4.1). Let $\mathcal{A}=\mathcal{Z D} \mathcal{Z}^{-1}$, where $\mathcal{D}$ is the diagonal matrix whose elements are the eigenvalues $\lambda_{1}, \ldots, \lambda_{n+m}$ and $\mathcal{Z}$ is the eigenvector matrix.

THEOREM 4.5 ([4]). Let the initial residual $R_{0}$ be decomposed as $R_{0}=\mathcal{Z} \beta$, where $\beta$ is an $(n+m) \times$ s matrix whose columns are denoted by $\beta^{(1)}, \ldots, \beta^{(s)}$. Let $R_{k}=\mathcal{B}-\mathcal{A} \mathcal{X}_{k}$ be the $k$ th residual obtained by the global GMRES method when applied to (1.1). Then we have

$$
\left\|R_{k}\right\|_{F}^{2} \leq \frac{\|\mathcal{Z}\|_{2}^{2}}{e_{1}^{T}\left(V_{k+1}^{T} \widetilde{\mathcal{D}} V_{k+1}\right)^{-1} e_{1}}
$$

where

$$
\widetilde{\mathcal{D}}=\left[\begin{array}{ccc}
\sum_{i=1}^{s}\left|\beta_{1}{ }^{(i)}\right|^{2} & & \\
& \ddots & \\
& & \sum_{i=1}^{s}\left|\beta_{n+m}{ }^{(i)}\right|^{2}
\end{array}\right] \text { and } V_{k+1}=\left[\begin{array}{cccc}
1 & \lambda_{1} & \ldots & \lambda_{1}^{k} \\
\vdots & \vdots & & \vdots \\
1 & \lambda_{n+m} & \ldots & \lambda_{n+m}^{k}
\end{array}\right] \text {. }
$$

The coefficients $\beta_{1}^{(i)}, \ldots, \beta_{n+m}^{(i)}$ are the components of the vector $\beta^{(i)}$, and $e_{1}$ is the first unit vector of $\mathbb{R}^{k+1}$.

5. Preconditioning. In this section we investigate a regularization preconditioner for solving saddle point problems with multiple right-hand sides (1.1), where $A \in \mathbb{R}^{n \times n}$ is a symmetric large, sparse, and invertible matrix and $B \in \mathbb{R}^{m \times n}$ has full row rank. The idea of preconditioning is to transform the linear system (1.1) into another one that is easier to solve. Left preconditioning of (1.1) gives the following new linear system

$$
\mathcal{M}^{-1} \mathcal{A X}=\mathcal{M}^{-1} \mathcal{B} .
$$

Although right preconditioning can be used in our context, we will focus only on left preconditioning throughout this work. By exploiting the particular block structure of (1.1), several block preconditioners have been derived [6]. We introduce and analyze the matrix block preconditioner for (1.1) defined by

$$
\mathcal{P}_{\epsilon, \alpha, Q}=\left[\begin{array}{cc}
A & B^{T} \\
\epsilon B & \alpha Q
\end{array}\right],
$$

with $\alpha>0$ and $Q$ a symmetric and positive definite matrix. For example, for the discrete Stokes system, $Q$ may be an approximation of the pressure Schur complement $S=B A^{-1} B^{T}$; see [12]. 


\section{ETNA}

Kent State University and

Johann Radon Institute (RICAM)

5.1. Properties of the preconditioner $\mathcal{P}_{\epsilon, \alpha, Q}$. We describe a block factorization and some properties of the preconditioner $\mathcal{P}_{\epsilon, \alpha, Q}$.

PROPOSITION 5.1. The preconditioner (5.1) has the block-triangular factorization

$$
\mathcal{P}_{\epsilon, \alpha, Q}=\left[\begin{array}{cc}
A & B^{T} \\
\epsilon B & \alpha Q
\end{array}\right]=\left[\begin{array}{cc}
I & O \\
\epsilon B A^{-1} & I
\end{array}\right]\left[\begin{array}{cc}
A & O \\
O & \tilde{S}
\end{array}\right]\left[\begin{array}{cc}
I & A^{-1} B^{T} \\
O & I
\end{array}\right],
$$

where $\tilde{S}=\left(\alpha Q-\epsilon B A^{-1} B^{T}\right)$. If $\epsilon=-1$, or $\epsilon=1$ and $\alpha \lambda_{\min }(Q)>\lambda_{\max }\left(B A^{-1} B^{T}\right)$, then the block $\tilde{S}$ is a positive definite matrix.

The inverse of the preconditioner $\mathcal{P}_{\epsilon, \alpha, Q}$ is given by

$$
\mathcal{P}_{\epsilon, \alpha, Q}^{-1}=\left[\begin{array}{cc}
A & B^{T} \\
\epsilon B & \alpha Q
\end{array}\right]^{-1}=\left[\begin{array}{cc}
I & -A^{-1} B^{T} \\
O & I
\end{array}\right]\left[\begin{array}{cc}
A^{-1} & O \\
O & \tilde{S}^{-1}
\end{array}\right]\left[\begin{array}{cc}
I & O \\
B A^{-1} & I
\end{array}\right] .
$$

The eigenvalue and eigenvector distributions of the preconditioned matrix relate closely to the convergence rate of Krylov subspace methods. Therefore, it is meaningful to investigate the spectral properties of the preconditioned matrix $\mathcal{P}_{\epsilon, \alpha, Q}^{-1} \mathcal{A}$. In the following theorem, we will deduce the eigenvalue distribution of $\mathcal{P}_{\epsilon, \alpha, Q}^{-1} \mathcal{A}$.

THEOREM 5.2. Let the preconditioner $\mathcal{P}_{\epsilon, \alpha, Q}$ be defined as in (5.1) with $A$ and $Q$ positive definite matrices. Then the matrix $\mathcal{P}_{\epsilon, \alpha, Q}^{-1} \mathcal{A}$ is diagonalizable and has $m_{1}+1$ distinct eigenvalues $\left\{1, \lambda_{1}, \ldots, \lambda_{m_{1}}\right\}$, with $1 \leq m_{1} \leq m$.

Proof. Let $\lambda$ be an eigenvalue of the preconditioned matrix $\mathcal{P}_{\epsilon, \alpha, Q}^{-1} \mathcal{A}$ and $\left(x^{T}, y^{T}\right)^{T}$ be the corresponding eigenvector. To derive the eigenvalue distribution, we consider the following generalized eigenvalue problem:

$$
\mathcal{P}_{\epsilon, \alpha, Q}^{-1} \mathcal{A}\left[\begin{array}{l}
x \\
y
\end{array}\right]=\left[\begin{array}{cc}
I_{n} & K_{1} \\
0 & K_{2}
\end{array}\right]\left[\begin{array}{l}
x \\
y
\end{array}\right]=\lambda\left[\begin{array}{l}
x \\
y
\end{array}\right]
$$

where

$$
\tilde{S}=\left(\alpha Q-\epsilon B A^{-1} B^{T}\right), K_{1}=\left(I+\epsilon A^{-1} B^{T} \tilde{S}^{-1} B\right) A^{-1} B^{T}, K_{2}=-\epsilon \tilde{S}^{-1} B A^{-1} B^{T} .
$$

Equation (5.2) can be written as

$$
\left\{\begin{array}{l}
(1-\lambda) x=-\left(I+\epsilon A^{-1} B^{T} \tilde{S}^{-1} B\right) A^{-1} B^{T} y \\
B A^{-1} B^{T} y=\frac{\alpha \lambda}{\epsilon(\lambda-1)} Q y .
\end{array}\right.
$$

If $\lambda=1$ holds true, then from the first equation of (5.3), we easily get

$$
\left(I+\epsilon A^{-1} B^{T} \tilde{S}^{-1} B\right) A^{-1} B^{T} y=0 .
$$

When $y=0$, equation (5.3) is always true when $\lambda=1$. Thus, there are $n$ linearly independent eigenvectors $\left[\begin{array}{c}u^{(i)} \\ 0\end{array}\right], i=1, \ldots, n$, corresponding to the eigenvalue 1 , where $u^{(i)}$ are arbitrary linearly independent vectors. If $\lambda \neq 1$ and $y=0$, then we obtain from the first equation of (5.3) that $x=0$. This contradicts the assumption that $\left(x^{T}, y^{T}\right)^{T}$ is an eigenvector of the preconditioned matrix $\mathcal{P}_{\epsilon, \alpha, Q}^{-1} \mathcal{A}$, and therefore $y \neq 0$. If $y$ satisfies the second equation of (5.3), then

$$
B A^{-1} B^{T} y=\mu Q y
$$


where

$$
\mu=\frac{\epsilon \alpha \lambda}{(\lambda-1)}
$$

We deduce that

$$
\lambda=\frac{\mu}{\mu-\alpha \epsilon} .
$$

Since the matrix $Q$ is positive definite, it admits a Cholesky decomposition $Q=R^{T} R$. Premultiplying (5.4) with $R^{-T}$ results in

$$
R^{-T} B A^{-1} B^{T} R^{-1} z=\mu z \quad \text { with } z=R y .
$$

Since $R^{-T} B A^{-1} B^{T} R^{-1}$ is a positive definite matrix, there are $m$ linearly independent eigenvectors of the form $w^{(i)}=R^{-1} z^{(i)}, i=1, \ldots, m$, where $z^{(i)}$ are orthogonal eigenvectors of $R^{-T} B A^{-1} B^{T} R^{-1}$.

Let $U, V$, and $W$ be the matrices whose columns are $\left(u^{(1)}, \ldots, u^{(n)}\right),\left(v^{(1)}, \ldots, v^{(m)}\right)$, and $\left(w^{(1)}, \ldots, w^{(m)}\right)$, respectively, where

$$
v^{(i)}=\frac{\left(I+\epsilon A^{-1} B^{T} \tilde{S}^{-1} B\right) A^{-1} B^{T}}{(\lambda-1)} w^{(i)}, \quad i=1, \ldots, m .
$$

Then $\mathcal{Z}=\left[\begin{array}{cc}U & V \\ O & W\end{array}\right]$ is a matrix of eigenvectors of $\mathcal{P}_{\epsilon, \alpha, Q}^{-1} \mathcal{A}$. Moreover, since $U$ and $W$ are nonsingular matrices, $\mathcal{Z}$ is also nonsingular. Consequently, $\mathcal{P}_{\epsilon, \alpha, Q}^{-1} \mathcal{A}$ is diagonalizable.

REMARK 5.3.

1. When the hypotheses of the preceding theorem are satisfied, the preconditioned global Krylov subspace methods converge in at most $m_{1}+1$ iterations. Moreover, in Theorem 4.5, we can see that the bound depends only on the distribution of the eigenvalues $\left\{1, \lambda_{1}, \ldots, \lambda_{m_{1}}\right\}$ and on the matrix $\mathcal{Z}$.

2. If $A$ is positive definite, $\epsilon=-1$, and $\mu$ satisfies the generalized eigenvalue problem (5.4), then

$$
\mu=\frac{\lambda \alpha}{(1-\lambda)} \quad \text { and } \quad \lambda=\frac{\mu}{\alpha+\mu} \leq 1
$$

Hence when $\alpha \rightarrow 0, \lambda \rightarrow 1$.

For the discrete Stokes system, if $\gamma$ is the (generalized) inf-sup constant and $\Gamma$ is a boundedness constant, then we have $\gamma^{2} \leq \mu \leq \Gamma^{2}$ (see for example [12, p. 173]) and

$$
\lambda \in\left[\frac{\gamma^{2}}{\gamma^{2}+\alpha}, \frac{\Gamma^{2}}{\Gamma^{2}+\alpha}\right] .
$$

5.2. Preconditioned global subspace Krylov solvers. We consider now preconditioned global Krylov subspace methods for solving

$$
\mathcal{P}_{\epsilon, \alpha, Q}^{-1} \mathcal{A X}=\mathcal{P}_{\epsilon, \alpha, Q}^{-1} \mathcal{B}
$$

Each global Krylov iteration requires the solution of a linear system of the form

$$
\underbrace{\left[\begin{array}{cc}
A & B^{T} \\
\epsilon B & \alpha Q
\end{array}\right]}_{\mathcal{P}_{\epsilon, \alpha, Q}}\left[\begin{array}{l}
Z_{1} \\
Z_{2}
\end{array}\right]=\left[\begin{array}{l}
V_{1} \\
V_{2}
\end{array}\right],
$$



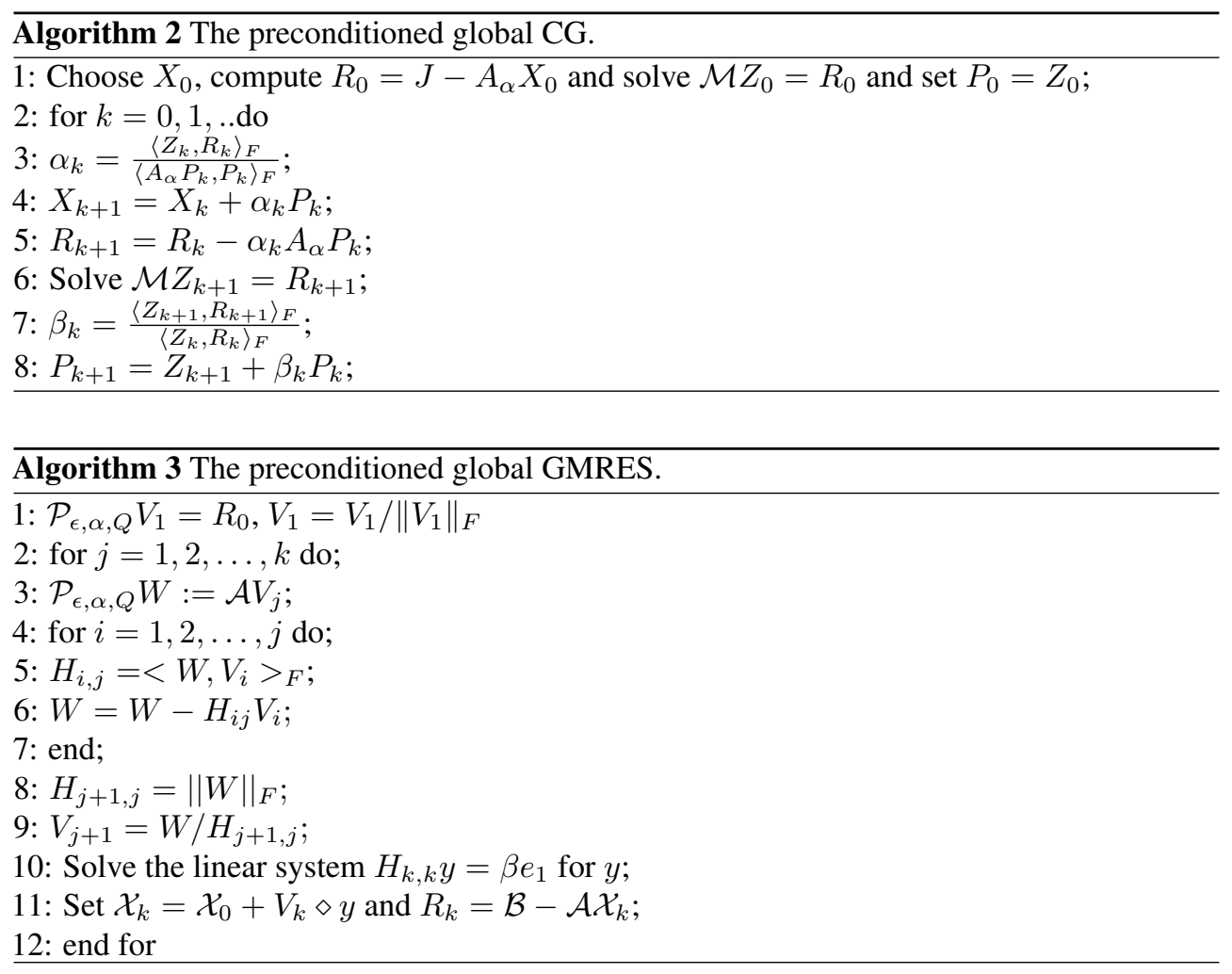

with $V_{1} \in \mathbb{R}^{n \times s}, V_{2} \in \mathbb{R}^{m \times s}, Z_{1} \in \mathbb{R}^{n \times s}$, and $Z_{2} \in \mathbb{R}^{m \times s}$. This system can be solved in two stages as:

$$
\begin{aligned}
\underbrace{\left(A-\frac{\epsilon}{\alpha} B^{T} Q^{-1} B\right)}_{A_{\alpha}} Z_{1} & =\underbrace{V_{1}-\frac{1}{\alpha} B^{T} Q^{-1} V_{2}}_{J}, \\
Z_{2} & =\frac{1}{\alpha} Q^{-1}\left(V_{2}-\epsilon B Z_{1}\right) .
\end{aligned}
$$

The system $A_{\alpha} Z_{1}=J$ is solvable if the symmetric matrix $A_{\alpha}$ is invertible. If $\epsilon=-1$, then the matrix $A_{\alpha}$ is symmetric positive definite. We would like to solve the preconditioned system

$$
\mathcal{M}^{-1} A_{\alpha}=\mathcal{M}^{-1} J
$$

where $\mathcal{M}$ is a preconditioner. Depending on the value of $\epsilon$, the matrix of the linear system (1.1) or the preconditioner may be symmetric or nonsymmetric. When the matrix is symmetric positive definite, we apply the preconditioned global CG method [15], Algorithm 2. If $\epsilon=1$, then the matrix of (1.1) is symmetric indefinite, and we will use the global MINRES method [10]. When the matrix of the system is nonsymmetric, several global subspace Krylov methods can be used. The most useful one is the global GMRES method, Algorithm 3, which computes iterates that minimise the Frobenius norm of the residual. We will define here a "flexible" version of global GMRES, Algorithm 4, which allows for a preconditioner that varies during the iterations. The standard Flexible GMRES was defined by Saad [16]. Since 

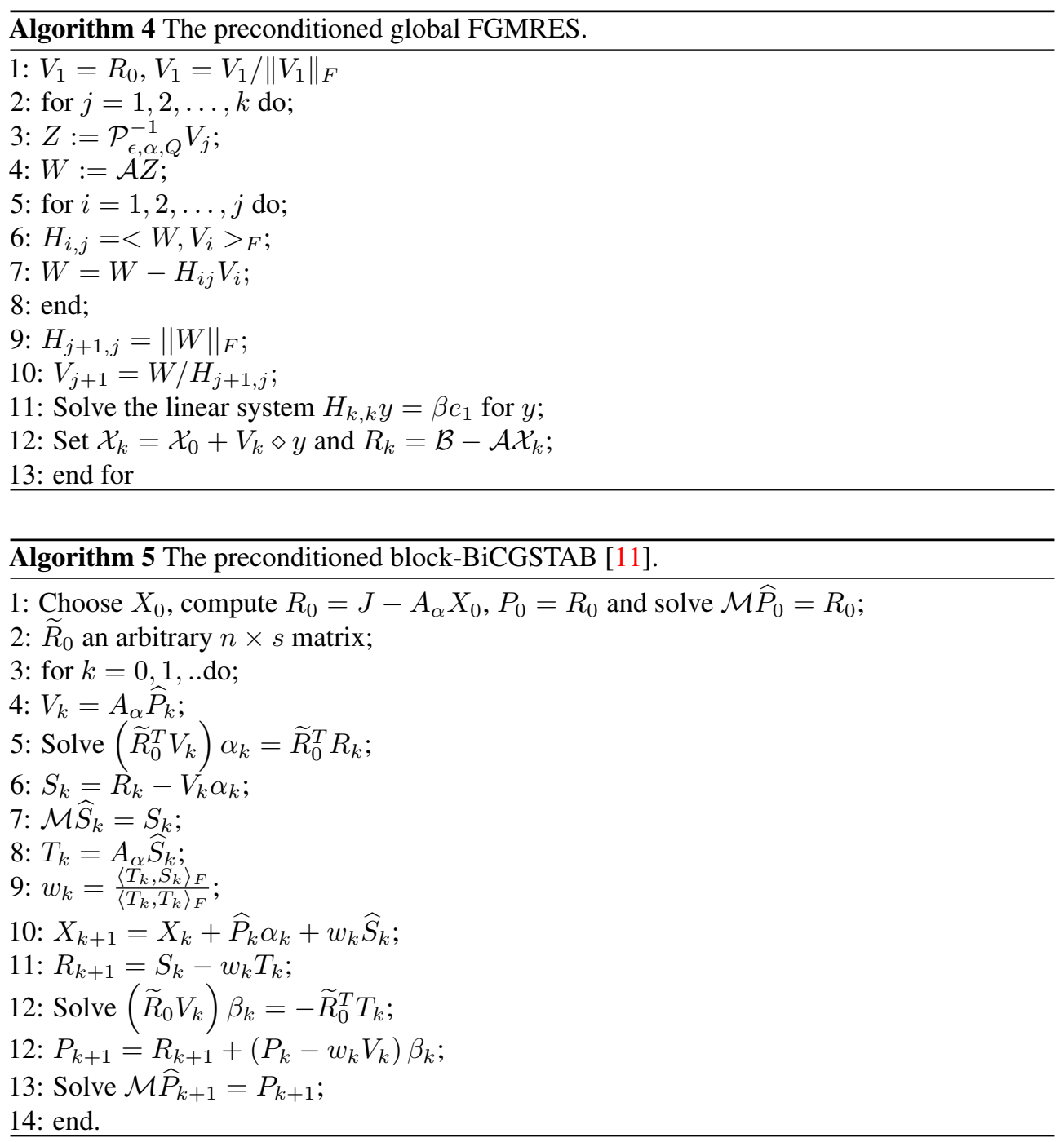

the amount of computation and storage is increasing with each iteration of the global GMRES and global FGMRES methods, we will also consider the block BiCGSTAB method introduced in [11], Algorithm 5. The latter requires only a fixed amount of work per iteration.

6. Numerical experiments. In this section we present the results of numerical experiments that illustrate the convergence behavior of the preconditioned global GMRES, global MINRES, and global FGMRES methods using the proposed preconditioner. All of the reported numerical results were performed on a 64-bit $2.49 \mathrm{GHz}$ core i5 processor and 8.00 GB RAM using MATLAB 2016. In all the experiments, we used ten right-hand sides. CPU times and iteration counts are reported in the rows "CPU" and "Iter" in the tables below. In the cases of the preconditioned global GMRES and global FGMRES methods, two values are reported at "Iter", namely, the number of steps of the preconditioned global GMRES method or of the preconditioned global FGMRES method and in parenthesis the total number of inner preconditioned conjugate gradient iterations. In all the tables, a dagger " $\nmid$ " indicates that the method has not converged in at most 500 iterations. 


\section{ETNA}

Kent State University and

Johann Radon Institute (RICAM)

TABLE 6.1

The size of the matrices $A$ and $B$ for the lid driven cavity problem on $2^{l} \times 2^{l}$.

Lid driven cavity

\begin{tabular}{c|c|c|c|c|c}
$l$ & $n$ & $m$ & size of $A$ & size of $B$ & size of $Q$ \\
\hline 4 & 578 & 192 & $578 \times 578$ & $578 \times 192$ & $192 \times 192$ \\
5 & 2178 & 768 & $2178 \times 2178$ & $2178 \times 766$ & $766 \times 766$ \\
6 & 8450 & 3070 & $8450 \times 8450$ & $8450 \times 3070$ & $3070 \times 3070$ \\
7 & 33282 & 12288 & $33282 \times 33282$ & $33282 \times 12288$ & $12288 \times 12288$
\end{tabular}

When $\epsilon=-1$, we used the preconditioned global GMRES and global FGMRES methods to solve the nonsymmetric saddle point problems with multiple right-hand sides (1.1). In practice, the preconditioners used for solving (1.1) are $\mathcal{P}_{\epsilon, \alpha, Q}, \mathcal{P}_{T}$, and $\mathcal{P}_{D}$, where $\mathcal{P}_{\epsilon, \alpha, Q}$, $\mathcal{P}_{T}$, and $\mathcal{P}_{D}$ are given as follows:

$$
\mathcal{P}_{\epsilon, \alpha, Q}=\left[\begin{array}{cc}
A & B^{T} \\
-B & \alpha Q
\end{array}\right], \quad \mathcal{P}_{T}=\left[\begin{array}{cc}
A & O \\
-B & S
\end{array}\right], \quad \text { and } \quad \mathcal{P}_{D}=\left[\begin{array}{cc}
A & O \\
O & S
\end{array}\right]
$$

When $\epsilon=1$, we used the preconditioned global MINRES methods to solve the symmetric saddle point problems with multiple right-hand sides (1.1). The preconditioners are given as follows:

$$
\mathcal{P}_{\epsilon, \alpha, Q}=\left[\begin{array}{ll}
A & B^{T} \\
B & \alpha Q
\end{array}\right] \text { and } \mathcal{P}_{D}=\left[\begin{array}{cc}
A & O \\
O & S
\end{array}\right]
$$

Here $S$ is a sparse approximation of the pressure Schur complement $B A^{-1} B^{T}$, and $Q$ is one of the matrices $I$ or $\operatorname{diag}(S)$. The parameter of the $\mathcal{P}_{\epsilon, \alpha, Q}$ preconditioner is chosen as $\alpha \in\left[10^{-5}, 1\right]$. In all the numerical tests below, the initial guess is taken to be the null matrix, and the right-hand side $\mathcal{B} \in \mathbb{R}^{(n+m) \times s}$ is chosen such that the exact solution of the saddle point problem (1.1) is a matrix of ones. For the preconditioned global MINRES, global GMRES, and global Flexible GMRES (FGMRES) methods, the iterations were stopped where

$$
\frac{\left\|\mathcal{P}^{-1} \mathcal{B}-\mathcal{P}^{-1} \mathcal{A} \mathcal{X}^{(k)}\right\|_{F}}{\left\|\mathcal{P}^{-1} \mathcal{B}\right\|_{F}}<10^{-12}
$$

where $\mathcal{P}$ is one of the preconditioners $\mathcal{P}_{\epsilon, \alpha, Q}, \mathcal{P}_{T}$, or $\mathcal{P}_{D}$. Here $\|\cdot\|_{F}$ stands for the Frobenius norm, and $\mathcal{X}^{(k)} \in \mathbb{R}^{(n+m) \times s}$ denotes the current iterate. When using Algorithm 2 for solving the first system of Algorithm 4, the preconditioner used is a drop tolerance-based incomplete Cholesky factorization computed using the Matlab function "ichol(.,opts)", where

- opts.type = 'ict',

- opts.droptol = 1e-2.

The inner relative residual norm is less than tol $\left(=10^{-9}\right)$.

We use the IFISS software package developed by Elman et al. [12] to generate the linear systems with multiple right-hand sides corresponding to $l=4, l=5, l=6$, and $l=7$. The IFISS software provides the matrices Ast, Bst, and $Q$ for the matrices $A, B$, and $S$, respectively. For the lid driven cavity problem, Bst is a rank deficient matrix, thus we drop the first two rows of $B$ st to get a full rank matrix.

Generic information of the test problems, including $n$ and $m$, are provided in Table 6.1. Numerical results for the nonsymmetric saddle point problem with multiple right-hand sides $(\epsilon=-1)$ are presented in Table 6.2. In Tables 6.2, 6.3, 6.4, and 6.5 we report the results for the preconditioned global GMRES and global FGMRES iterative methods. From the numerical results listed in the tables, we can conclude that the $\mathcal{P}_{\epsilon, \alpha, Q}$ preconditioned global 


\section{ETNA}

Kent State University and

Johann Radon Institute (RICAM)

TABLE 6.2

Numerical results for the preconditioner $\mathcal{P}_{\epsilon, \alpha, Q}$ with $Q=I$.

\begin{tabular}{c|c|l|l|l} 
& $l$ & $l=5$ & $l=6$ & $l=7$ \\
\hline$\alpha$ & & global GMRES & global GMRES & global GMRES \\
\hline $10^{-5}$ & Iter & $6(103)$ & $7(155)$ & $9(225)$ \\
& CPU & 1.17 & 7.31 & 89.29 \\
& RES & $2.93 \mathrm{e}-05$ & $2.11 \mathrm{e}-04$ & $1.39 \mathrm{e}-07$ \\
& ERR & $1.65 \mathrm{e}-03$ & $1.20 \mathrm{e}-02$ & $1.92 \mathrm{e}-05$ \\
\hline $10^{-4}$ & Iter & $8(81)$ & $11(100)$ & $17(116)$ \\
& CPU & 1.25 & 7.43 & 81.65 \\
& RES & $3.93 \mathrm{e}-06$ & $6.47 \mathrm{e}-07$ & $1.54 \mathrm{e}-07$ \\
& ERR & $3.43 \mathrm{e}-05$ & $1.39 \mathrm{e}-05$ & $1.09 \mathrm{e}-05$ \\
\hline $10^{-3}$ & Iter & $14(47)$ & $21(46)$ & $32(67)$ \\
& CPU & 1.28 & 7.79 & 102.83 \\
& RES & $1.17 \mathrm{e}-07$ & $4.90 \mathrm{e}-08$ & $7.66 \mathrm{e}-08$ \\
& ERR & $1.79 \mathrm{e}-06$ & $9.18 \mathrm{e}-07$ & $5.47 \mathrm{e}-06$ \\
\hline $10^{-2}$ & Iter & $27(23)$ & $37(33)$ & $43(55)$ \\
& CPU & 1.50 & 9.28 & 94.54 \\
& RES & $1.18 \mathrm{e}-08$ & $1.46 \mathrm{e}-08$ & $2.30 \mathrm{e}-08$ \\
& ERR & $1.69 \mathrm{e}-07$ & $5.62 \mathrm{e}-07$ & $2.73 \mathrm{e}-06$ \\
\hline $10^{-1}$ & Iter & $40(17)$ & $44(29)$ & $46(50)$ \\
& CPU & 1.53 & 9.43 & 97.17 \\
& RES & $5.43 \mathrm{e}-08$ & $1.97 \mathrm{e}-08$ & $2.18 \mathrm{e}-08$ \\
& ERR & $1.18 \mathrm{e}-06$ & $2.88 \mathrm{e}-06$ & $1.87 \mathrm{e}-06$ \\
\hline 1 & Iter & $42(16)$ & $45(26)$ & $46(50)$ \\
& CPU & 1.52 & 8.84 & 101.96 \\
& RES & $1.10 \mathrm{e}-08$ & $2.29 \mathrm{e}-08$ & $1.01 \mathrm{e}-08$ \\
& ERR & $3.37 \mathrm{e}-07$ & $9.37 \mathrm{e}-07$ & $2.80 \mathrm{e}-06$
\end{tabular}

GMRES and global FGMRES methods require less iterations and less CPU time than $\mathcal{P}_{T}$ and $\mathcal{P}_{D}$ in all trials.

Table 6.6 indicates that the $\mathcal{P}_{\epsilon, \alpha, Q}$ preconditioner with $Q=I$ leads to much better numerical results than the $\mathcal{P}_{\epsilon, \alpha, Q}$ preconditioner with $Q=\operatorname{diag}(S)$ and $Q=S$, as the $\mathcal{P}_{\epsilon, \alpha, Q}$ preconditioned GMRES method with $Q=I$ need less CPU times in all trials and iteration steps for some values of $\alpha$ compared with the other choices of the matrix $Q$.

The numerical results for the symmetric saddle point problem with multiple right-hand sides $(\epsilon=1)$ are given in Table 6.7. As observed in Table 6.7 and 6.8, the $\mathcal{P}_{\epsilon, \alpha, Q}$ preconditioned global MINRES method with the proper parameter $\alpha$ has a better performance than the $\mathcal{P}_{D}$ preconditioned global MINRES method in terms of the iterations and CPU times.

Numerical results for the nonsymmetric saddle point problem with multiple right-hand sides $(\epsilon=-1)$ and when the discretization is non-uniform are provided in Table 6.9. For $\alpha=10^{-5}$ and $\alpha=10^{-4}$, the $\mathcal{P}_{\epsilon, \alpha, Q}$ (inner block-BiCGSTAB) preconditioned global GMRES method diverge while the $\mathcal{P}_{\epsilon, \alpha, Q}$ (inner PCG) preconditioned global GMRES and the $\mathcal{P}_{\epsilon, \alpha, Q}$ (inner PCG) preconditioned global FGMRES converge.

For the following experiments we will illustrate the good behavior of the preconditioned global GMRES methods even for a particular right-hand sides. Numerical result for the preconditioned global GMRES methods with the right-hand sides $\mathcal{B}=\left[\begin{array}{l}F \\ O\end{array}\right]$ are given in 
TABLE 6.3

Numerical results for the preconditioner $\mathcal{P}_{\epsilon, \alpha, Q}$ with $Q=I$.

\begin{tabular}{c|c|l|l|l} 
& $l$ & $l=5$ & $l=6$ & $l=7$ \\
\hline$\alpha$ & & global FGMRES & global FGMRES & global FGMRES \\
\hline $10^{-5}$ & Iter & $6(85)$ & $7(143)$ & $8(206)$ \\
& CPU & 0.93 & 6.61 & 60.67 \\
& RES & $5.97 \mathrm{e}-04$ & $2.09 \mathrm{e}-04$ & $1.31 \mathrm{e}-07$ \\
& ERR & $4.87 \mathrm{e}-03$ & $4.11 \mathrm{e}-03$ & $2.87 \mathrm{e}-05$ \\
\hline $10^{-4}$ & Iter & $7(69)$ & $10(90)$ & $14(106)$ \\
& CPU & 0.99 & 5.59 & 55.58 \\
& RES & $3.77 \mathrm{e}-06$ & $1.70 \mathrm{e}-07$ & $8.43 \mathrm{e}-08$ \\
& ERR & $3.23 \mathrm{e}-05$ & $2.55 \mathrm{e}-06$ & $2.46 \mathrm{e}-06$ \\
\hline $10^{-3}$ & Iter & $12(43)$ & $18(42)$ & $27(65)$ \\
& CPU & 0.98 & 6.99 & 68.58 \\
& RES & $1.08 \mathrm{e}-07$ & $6.30 \mathrm{e}-08$ & $7.05 \mathrm{e}-08$ \\
& ERR & $6.68 \mathrm{e}-07$ & $6.62 \mathrm{e}-07$ & $1.87 \mathrm{e}-06$ \\
\hline $10^{-2}$ & Iter & $24(21)$ & $33(32)$ & $39(53)$ \\
& CPU & 1.19 & 7.92 & 78.40 \\
& RES & $6.94 \mathrm{e}-08$ & $4.80 \mathrm{e}-08$ & $9.28 \mathrm{e}-08$ \\
& ERR & $2.01 \mathrm{e}-07$ & $4.76 \mathrm{e}-07$ & $3.57 \mathrm{e}-06$ \\
\hline \multirow{2}{*}{$10^{-1}$} & Iter & $37(16)$ & $41(28)$ & $45(51)$ \\
& CPU & 1.45 & 8.84 & 90.95 \\
& RES & $5.54 \mathrm{e}-08$ & $9.23 \mathrm{e}-08$ & $1.08 \mathrm{e}-07$ \\
& ERR & $1.09 \mathrm{e}-07$ & $1.12 \mathrm{e}-06$ & $2.04 \mathrm{e}-06$ \\
\hline 1 & Iter & $43(16)$ & $46(27)$ & $49(49)$ \\
& CPU & 1.66 & 9.04 & 96.55 \\
& RES & $7.39 \mathrm{e}-08$ & $7.92 \mathrm{e}-08$ & $1.08 \mathrm{e}-07$ \\
& ERR & $2.51 \mathrm{e}-07$ & $1.78 \mathrm{e}-06$ & $1.27 \mathrm{e}-06$
\end{tabular}

TABLE 6.4

Numerical results for the three preconditioned global GMRES methods.

\begin{tabular}{|c|c|c|c|c|}
\hline$l$ & & $\begin{array}{c}\mathcal{P}_{\epsilon, \alpha, Q} \\
\left(\alpha=10^{-4}, Q=I\right)\end{array}$ & $\mathcal{P}_{T}$ & $\mathcal{P}_{D}$ \\
\hline$l=5$ & $\begin{array}{l}\text { Iter } \\
\text { CPU } \\
\text { RES } \\
\text { ERR }\end{array}$ & $\begin{array}{l}42(16) \\
1.52 \\
1.10 \mathrm{e}-08 \\
3.37 \mathrm{e}-07\end{array}$ & $\begin{array}{l}60(15) \\
2.24 \\
1.00 \mathrm{e}-07 \\
3.48 \mathrm{e}-06\end{array}$ & $\begin{array}{l}122(15) \\
4.90 \\
1.04 \mathrm{e}-06 \\
6.84 \mathrm{e}-06\end{array}$ \\
\hline$l=6$ & $\begin{array}{c}\text { Iter } \\
\text { CPU } \\
\text { RES } \\
\text { ERR }\end{array}$ & $\begin{array}{l}45(26) \\
8.84 \\
2.29 \mathrm{e}-08 \\
9.37 \mathrm{e}-07\end{array}$ & $\begin{array}{l}62(26) \\
11.06 \\
2.04 \mathrm{e}-07 \\
6.22 \mathrm{e}-06\end{array}$ & $\begin{array}{l}129(25) \\
22.70 \\
9.65 \mathrm{e}-07 \\
5.57 \mathrm{e}-05\end{array}$ \\
\hline$l=7$ & $\begin{array}{l}\text { Iter } \\
\text { CPU } \\
\text { RES } \\
\text { ERR }\end{array}$ & $\begin{array}{l}46(50) \\
101.96 \\
1.01 \mathrm{e}-08 \\
3.37 \mathrm{e}-07\end{array}$ & $\begin{array}{l}64(47) \\
114.56 \\
3.66 \mathrm{e}-07 \\
3.04 \mathrm{e}-05\end{array}$ & $\begin{array}{l}133(46) \\
295.49 \\
2.14 \mathrm{e}-06 \\
1.21 \mathrm{e}-04\end{array}$ \\
\hline
\end{tabular}


TABLE 6.5

Numerical results for the three preconditioned global FGMRES methods.

\begin{tabular}{|c|c|c|c|c|}
\hline$l$ & & $\begin{array}{c}\mathcal{P}_{\epsilon, \alpha, Q} \\
\left(\alpha=10^{-4}, Q=I\right)\end{array}$ & $\mathcal{P}_{T}$ & $\mathcal{P}_{D}$ \\
\hline$l=5$ & $\begin{array}{l}\text { Iter } \\
\text { CPU } \\
\text { RES } \\
\text { ERR }\end{array}$ & $\begin{array}{l}7(69) \\
0.99 \\
3.77 \mathrm{e}-06 \\
3.23 \mathrm{e}-05\end{array}$ & $\begin{array}{l}58(16) \\
2.24 \\
1.65 \mathrm{e}-06 \\
1.11 \mathrm{e}-05\end{array}$ & $\begin{array}{l}115(16) \\
3.788 .30 \mathrm{e}-07 \\
1.41 \mathrm{e}-06\end{array}$ \\
\hline$l=6$ & $\begin{array}{l}\text { Iter } \\
\text { CPU } \\
\text { RES } \\
\text { ERR }\end{array}$ & $\begin{array}{l}10(90) \\
5.59 \\
1.70 \mathrm{e}-07 \\
2.55 \mathrm{e}-06\end{array}$ & $\begin{array}{l}59(27) \\
11.62 \\
1.05 \mathrm{e}-07 \\
4.36 \mathrm{e}-06\end{array}$ & $\begin{array}{l}119(27) \\
22.99 \\
5.30 \mathrm{e}-08 \\
1.16 \mathrm{e}-06\end{array}$ \\
\hline$l=7$ & $\begin{array}{l}\text { Iter } \\
\text { CPU } \\
\text { RES } \\
\text { ERR }\end{array}$ & $\begin{array}{l}14(106) \\
55.58 \\
8.43 \mathrm{e}-08 \\
2.46 \mathrm{e}-06\end{array}$ & $\begin{array}{l}61(49) \\
92.52 \\
1.36 \mathrm{e}-07 \\
1.75 \mathrm{e}-05\end{array}$ & $\begin{array}{l}121(50) \\
181.65 \\
9.32 \mathrm{e}-08 \\
1.00 \mathrm{e}-06\end{array}$ \\
\hline
\end{tabular}

TABLE 6.6

Numerical results for the preconditioner $\mathcal{P}_{\epsilon, \alpha, Q}$ with $l=5$.

\begin{tabular}{c|c|l|l|l}
$\alpha$ & $Q$ & $Q=I$ & $Q=\operatorname{diag}(S)$ & $Q=S$ \\
\hline & & global GMRES & global GMRES & global GMRES \\
\hline $10^{-5}$ & Iter & $6(103)$ & $9(211)$ & $14(215)$ \\
& CPU & 1.17 & 3.45 & 6.05 \\
& RES & $1.25 \mathrm{e}-04$ & $8.11 \mathrm{e}-09$ & $1.62 \mathrm{e}-07$ \\
& ERR & $6.06 \mathrm{e}-03$ & $6.26 \mathrm{e}-08$ & $9.49 \mathrm{e}-07$ \\
\hline $10^{-4}$ & Iter & $8(81)$ & $8(190)$ & $15(209)$ \\
& CPU & 1.25 & 2.92 & 6.19 \\
& RES & $3.93 \mathrm{e}-06$ & $3.63 \mathrm{e}-09$ & $1.72 \mathrm{e}-07$ \\
& ERR & $3.43 \mathrm{e}-05$ & $2.59 \mathrm{e}-08$ & $9.91 \mathrm{e}-07$ \\
\hline $10^{-3}$ & Iter & $14(47)$ & $11(162)$ & $22(173)$ \\
& CPU & 1.28 & 3.62 & 7.51 \\
& RES & $1.17 \mathrm{e}-07$ & $4.16 \mathrm{e}-09$ & $8.08 \mathrm{e}-09$ \\
& ERR & $1.79 \mathrm{e}-06$ & $3.76 \mathrm{e}-08$ & $5.51 \mathrm{e}-08$ \\
\hline $10^{-2}$ & Iter & $27(23)$ & $14(123)$ & $33(123)$ \\
& CPU & 1.50 & 4.37 & 8.87 \\
& RES & $1.18 \mathrm{e}-08$ & $2.80 \mathrm{e}-08$ & $9.72 \mathrm{e}-08$ \\
& ERR & $1.69 \mathrm{e}-07$ & $1.77 \mathrm{e}-07$ & $6.21 \mathrm{e}-07$ \\
\hline \multirow{2}{*}{$10^{-1}$} & Iter & $40(17)$ & $23(65)$ & $115(65)$ \\
& CPU & 1.53 & 3.64 & 18.63 \\
& RES & $5.43 \mathrm{e}-08$ & $1.21 \mathrm{e}-07$ & $8.72 \mathrm{e}-08$ \\
& ERR & $1.18 \mathrm{e}-06$ & $8.72 \mathrm{e}-07$ & $5.36 \mathrm{e}-07$ \\
\hline \multirow{2}{*}{1} & Iter & $42(16)$ & $27(26)$ & $\dagger$ \\
& CPU & 1.52 & 1.73 & $\dagger$ \\
& RES & $1.10 \mathrm{e}-08$ & $1.43 \mathrm{e}-07$ & $\dagger$ \\
& ERR & $3.37 \mathrm{e}-07$ & $3.03 \mathrm{e}-06$ & $\dagger$
\end{tabular}


TABLE 6.7

Numerical results for the preconditioner $\mathcal{P}_{\epsilon, \alpha, Q}$ with $Q=I$.

\begin{tabular}{c|c|l|l|l} 
& & $l=5$ & $l=6$ & $l=7$ \\
\hline$\alpha$ & & global MINRES & global MINRES & global MINRES \\
\hline $10^{-1}$ & Iter & 38 & 38 & 34 \\
& CPU & 0.41 & 2.58 & 45.60 \\
& RES & $3.11 \mathrm{e}-10$ & $6.95 \mathrm{e}-10$ & $3.07 \mathrm{e}-08$ \\
& ERR & $1.91 \mathrm{e}-07$ & $5.16 \mathrm{e}-06$ & $7.02 \mathrm{e}-04$ \\
\hline \multirow{2}{*}{1} & Iter & 37 & 38 & 37 \\
& CPU & 0.36 & 2.41 & 46.18 \\
& RES & $1.00 \mathrm{e}-09$ & $1.90 \mathrm{e}-09$ & $5.46 \mathrm{e}-08$ \\
& ERR & $1.59 \mathrm{e}-06$ & $8.33 \mathrm{e}-05$ & $2.68 \mathrm{e}-03$ \\
\hline \multirow{2}{*}{10} & Iter & 39 & 38 & 38 \\
& CPU & 0.54 & 2.79 & 49.52 \\
& RES & $2.11 \mathrm{e}-09$ & $7.51 \mathrm{e}-09$ & $1.45 \mathrm{e}-07$ \\
& ERR & $8.05 \mathrm{e}-07$ & $9.25 \mathrm{e}-06$ & $1.70 \mathrm{e}-02$
\end{tabular}

TABLE 6.8

Numerical results for global MINRES with $\mathcal{P}_{\epsilon, \alpha, Q}$ and $\mathcal{P}_{D}$.

\begin{tabular}{|c|c|c|c|}
\hline$l$ & & $\begin{array}{c}\mathcal{P}_{\epsilon, \alpha, Q} \\
\left(\alpha=10^{-4}, Q=I\right)\end{array}$ & $\mathcal{P}_{D}$ \\
\hline \multirow[t]{4}{*}{$l=5$} & Iter & 37 & 104 \\
\hline & $\mathrm{CPU}$ & 0.36 & 0.97 \\
\hline & RES & $1.00 \mathrm{e}-09$ & $8.75 \mathrm{e}-10$ \\
\hline & ERR & $1.59 \mathrm{e}-06$ & $1.26 \mathrm{e}-06$ \\
\hline \multirow[t]{4}{*}{$l=6$} & Iter & 38 & 110 \\
\hline & $\mathrm{CPU}$ & 2.41 & 5.22 \\
\hline & RES & $1.90 \mathrm{e}-09$ & $5.68 \mathrm{e}-10$ \\
\hline & ERR & $8.33 \mathrm{e}-05$ & $1.24 \mathrm{e}-04$ \\
\hline \multirow[t]{4}{*}{$l=7$} & Iter & 37 & $\dagger$ \\
\hline & $\mathrm{CPU}$ & 46.18 & $\dagger$ \\
\hline & RES & $5.46 \mathrm{e}-08$ & $\dagger$ \\
\hline & ERR & $2.68 \mathrm{e}-03$ & $\dagger$ \\
\hline
\end{tabular}

Table 6.10. From the numerical results in this table, we observe that the $\mathcal{P}_{\epsilon, \alpha, Q}$ preconditioner is superior to the $\mathcal{P}_{T}$ and the $\mathcal{P}_{D}$ preconditioners in terms of iterations and CPU times.

Results for the preconditioned global GMRES and preconditioned block GMRES methods incorporated with the $\mathcal{P}_{\epsilon, \alpha, Q}$ preconditioners are listed in Tables 6.11 for different values of $\alpha$. From the numerical results in the table, we can conclude that the $\mathcal{P}_{\epsilon, \alpha, Q}$ preconditioned global GMRES method requires less iterations and less CPU time than the $\mathcal{P}_{\epsilon, \alpha, Q}$ preconditioned block GMRES method in all trials. In each case when all two methods produce solutions, the $\mathcal{P}_{\epsilon, \alpha, Q}$ preconditioned global GMRES method gives smaller relative residuals and errors than the $\mathcal{P}_{\epsilon, \alpha, Q}$ preconditioned block GMRES method.

Table 6.12 reports the number of iterations (Iter), CPU times, residual (RES), and error (ERR) of the tested $\mathcal{P}_{\epsilon, \alpha, Q}$ preconditioned global GMRES methods with respect to different values of $\alpha$. From Table 6.12, we observe that the $\mathcal{P}_{\epsilon, \alpha, Q}$ (inner PGCG) preconditioned global GMRES method outperforms the $\mathcal{P}_{\epsilon, \alpha, Q}$ (inner PBl-CG) preconditioned global GMRES method in terms of iterations and CPU times. 


\section{ETNA}

Kent State University and

Johann Radon Institute (RICAM)

PRECONDITIONED GLOBAL KRYLOV METHOD FOR SADDLE POINT PROBLEMS

TABLE 6.9

\begin{tabular}{c|c|l|l|l}
\multicolumn{5}{c}{ Numerical results for the preconditioner $\mathcal{P}_{\epsilon, \alpha, Q}$ with $Q=I$ and $l=5}$. \\
\multirow{5}{*}{$\alpha$} & & $\begin{array}{l}\text { global GMRES } \\
\text { (inner PCG) }\end{array}$ & $\begin{array}{l}\text { global GMRES } \\
\text { (inner block- } \\
\text { BiCGSTAB) }\end{array}$ & $\begin{array}{l}\text { global FGMRES } \\
\text { (inner PCG) }\end{array}$ \\
\hline $10^{-3}$ & Iter & $37(76)$ & $44(67)$ & $33(79)$ \\
& CPU & 5.43 & 21.96 & 4.67 \\
& RES & $3.49 \mathrm{e}-06$ & $1.47 \mathrm{e}-05$ & $1.78 \mathrm{e}-07$ \\
& ERR & $1.04 \mathrm{e}-05$ & $6.37 \mathrm{e}-05$ & $6.95 \mathrm{e}-07$ \\
\hline $10^{-2}$ & Iter & $99(32)$ & $118(19)$ & $93(32)$ \\
& CPU & 7.17 & 16.90 & 6.11 \\
& RES & $4.81 \mathrm{e}-07$ & $7.31 \mathrm{e}-05$ & $1.25 \mathrm{e}-07$ \\
& ERR & $2.71 \mathrm{e}-06$ & $6.01 \mathrm{e}-04$ & $2.91 \mathrm{e}-07$ \\
\hline $10^{-1}$ & Iter & $192(18)$ & $192(9)$ & $178(18)$ \\
& CPU & 12.19 & 16.81 & 9.20 \\
& RES & $2.88 \mathrm{e}-08$ & $5.42 \mathrm{e}-07$ & $9.77 \mathrm{e}-08$ \\
& ERR & $5.12 \mathrm{e}-07$ & $1.54 \mathrm{e}-05$ & $2.17 \mathrm{e}-07$ \\
\hline \multirow{2}{*}{1} & Iter & $271(4)$ & $251(9)$ & $227(3)$ \\
& CPU & 12.29 & 19.34 & 10.42 \\
& RES & $2.59 \mathrm{e}-12$ & $3.55 \mathrm{e}-09$ & $1.30 \mathrm{e}-11$ \\
& ERR & $1.63 \mathrm{e}-06$ & $9.42 \mathrm{e}-07$ & $3.54 \mathrm{e}-08$
\end{tabular}

TABLE 6.10

Numerical results for the three preconditioned global GMRES methods.

\begin{tabular}{|c|c|c|c|c|}
\hline$l$ & & $\begin{array}{c}\mathcal{P}_{\epsilon, \alpha, Q} \\
\left(\alpha=10^{-5}, Q=I\right)\end{array}$ & $\mathcal{P}_{T}$ & $\mathcal{P}_{D}$ \\
\hline$l=5$ & $\begin{array}{l}\text { Iter } \\
\text { CPU } \\
\text { RES }\end{array}$ & $\begin{array}{l}6(92) \\
1.00 \\
4.46 \mathrm{e}-08\end{array}$ & $\begin{array}{l}62(15) \\
2.04 \\
6.18 \mathrm{e}-08\end{array}$ & $\begin{array}{l}134(15) \\
4.11 \\
3.56 \mathrm{e}-08\end{array}$ \\
\hline$l=6$ & $\begin{array}{l}\text { Iter } \\
\text { CPU } \\
\text { RES }\end{array}$ & $\begin{array}{l}8(132) \\
7.04 \\
7.23 \mathrm{e}-07\end{array}$ & $\begin{array}{l}64(26) \\
10.35 \\
2.44 \mathrm{e}-07\end{array}$ & $\begin{array}{l}142(26) \\
23.00 \\
8.58 \mathrm{e}-08\end{array}$ \\
\hline$l=7$ & $\begin{array}{l}\text { Iter } \\
\text { CPU } \\
\text { RES }\end{array}$ & $\begin{array}{l}10(199) \\
69.69 \\
4.59 \mathrm{e}-07\end{array}$ & $\begin{array}{l}66(47) \\
127.88 \\
4.09 \mathrm{e}-07\end{array}$ & $\begin{array}{l}148(46) \\
297.82 \\
6.54 \mathrm{e}-08\end{array}$ \\
\hline
\end{tabular}

7. Conclusion. We have presented the global approach as a new strategy to solve saddle point problems with multiple right-hand sides. In addition, we introduced and studied the preconditioner $\mathcal{P}_{\epsilon, \alpha, Q}$. Tables 6.2, 6.3, 6.4, 6.5, 6.6, and 6.9 illustrate that the $\mathcal{P}_{\epsilon, \alpha, Q}$ preconditioner with suitable choices of the parameter $\alpha$ has a better performance compared to $\mathcal{P}_{T}$ and $\mathcal{P}_{D}$ in all trials. The results in Tables 6.7 and 6.8 reveal the efficiency of the $\mathcal{P}_{\epsilon, \alpha, Q}$ preconditioned global MINRES method for solving the symmetric saddle point problem with several right-hand sides. We conclude that our preconditioned global Krylov subspace methods are very powerful for solving saddle point problems with multiple right-hand sides (1.1). 


\section{ETNA}

Kent State University and

Johann Radon Institute (RICAM)

TABLE 6.11

Numerical results for the preconditioned global GMRES and preconditioned block GMRES methods with $l=5$ and $Q=I$.

\begin{tabular}{|c|c|c|c|}
\hline$\alpha$ & & $\begin{array}{c}\mathcal{P}_{\epsilon, \alpha, Q} \\
\text { global GMRES }\end{array}$ & $\mathcal{P}_{\epsilon, \alpha, Q}$ block GMRES \\
\hline \multirow[t]{4}{*}{$10^{-5}$} & Iter & $6(104)$ & 164 \\
\hline & $\mathrm{CPU}$ & 1.93 & 20.56 \\
\hline & RES & $2.93 e-05$ & $8.78 \mathrm{e}-01$ \\
\hline & ERR & $1.65 \mathrm{e}-03$ & $2.17 e+01$ \\
\hline \multirow[t]{4}{*}{$10^{-4}$} & Iter & $8(81)$ & 191 \\
\hline & CPU & 1.25 & 24.60 \\
\hline & RES & $3.93 \mathrm{e}-06$ & $1.20 \mathrm{e}-03$ \\
\hline & ERR & $3.41 \mathrm{e}-05$ & $7.50 \mathrm{e}-03$ \\
\hline \multirow[t]{4}{*}{$10^{-3}$} & Iter & $4(47)$ & 164 \\
\hline & $\mathrm{CPU}$ & 1.30 & 15.77 \\
\hline & RES & $1.17 \mathrm{e}-07$ & $1.49 \mathrm{e}-06$ \\
\hline & ERR & $1.79 e-06$ & $6.68 e-06$ \\
\hline \multirow[t]{4}{*}{$10^{-2}$} & Iter & $27(23)$ & 132 \\
\hline & $\mathrm{CPU}$ & 1.44 & 9.66 \\
\hline & RES & $1.18 \mathrm{e}-08$ & $2.10 \mathrm{e}-08$ \\
\hline & ERR & $1.69 \mathrm{e}-07$ & $1.36 \mathrm{e}-07$ \\
\hline \multirow[t]{4}{*}{$10^{-1}$} & Iter & $40(17)$ & 171 \\
\hline & $\mathrm{CPU}$ & 1.54 & 18.17 \\
\hline & RES & $5.43 e-08$ & $4.74 \mathrm{e}-09$ \\
\hline & ERR & $1.18 \mathrm{e}-06$ & $4.68 \mathrm{e}-08$ \\
\hline \multirow[t]{4}{*}{1} & Iter & $42(16)$ & $236(42)$ \\
\hline & $\mathrm{CPU}$ & 1.66 & 41.95 \\
\hline & RES & $1.10 \mathrm{e}-08$ & $3.87 \mathrm{e}-09$ \\
\hline & ERR & $3.37 \mathrm{e}-07$ & $3.41 \mathrm{e}-07$ \\
\hline
\end{tabular}

\section{REFERENCES}

[1] W. E. ARNOLDI, The principle of minimized iteration in the solution of the matrix eigenvalue problem, Quart. Appl. Math., 9 (1951), pp. 17-29.

[2] Z.-Z. BAI AND M. BENZI, Regularized HSS iteration methods for saddle-point linear systems, BIT, 57 (2017), pp. 287-311.

[3] Z.-Z. BAI, G. H. Golub, AND J.-Y. PAn, Preconditioned Hermitian and skew-Hermitian splitting methods for non-Hermitian positive semidefinite linear systems, Numer. Math., 98 (2004), pp. 1-32.

[4] M. BellaliJ, K. JBilou, AND H. SADOK, New convergence results on the global GMRES method for diagonalizable matrices, J. Comput. Appl. Math., 219 (2008), pp. 350-358.

[5] P. BenNer, J. SAAK, M. StOLL, AND H. K. WeichSElt, Efficient solution of large-scale saddle point systems arising in Riccati-based boundary feedback stabilization of incompressible Stokes flow, SIAM J. Sci. Comput., 35 (2013), pp. S150-S170.

[6] M. BenZI, G. H. Golub, AND J. LiesEN, Numerical solution of saddle point problems, Acta Numer., 14 (2005), pp. 1-137.

[7] M. BENZI AND A. J. WATHEN, Some preconditioning techniques for saddle point problems, in Model Order Reduction: Theory, Research Aspects and Applications, W. H. A. Schilders, H A. van der Vorst, and J. Rommes, eds., vol. 13 of Math. Ind., Springer, Berlin, 2008, pp. 195-211.

[8] A. Bissuel, G. Allaire, L. Daumas, F. Chalot, and M. Mallet, Linear systems with multiple right-hand sides with GMRES: an application to aircraft design, in ECCOMAS Congress 2016, M. Papadrakakis, V. Papadopoulos, G. Stefanou, V. Plevris, eds., NTUA, Athens, 2016, pp. 7358-7371.

[9] R. Bouyouli, K. JBILOU, R. SADAKA, AND H. SAdOK, Convergence proprieties of some block Krylov 
ETNA

Kent State University and

Johann Radon Institute (RICAM)

PRECONDITIONED GLOBAL KRYLOV METHOD FOR SADDLE POINT PROBLEMS

TABLE 6.12

Numerical results for preconditioned global GMRES with $Q=I$ and $l=5$.

\begin{tabular}{|c|c|c|c|}
\hline$\alpha$ & & $\begin{array}{c}\mathcal{P}_{\epsilon, \alpha, Q} \\
\text { (inner PGCG) }\end{array}$ & $\begin{array}{c}\mathcal{P}_{\epsilon, \alpha, Q} \\
\text { (inner PBl-CG) }\end{array}$ \\
\hline \multirow[t]{4}{*}{$10^{-5}$} & Iter & $6(104)$ & $6(279)$ \\
\hline & CPU & 1.93 & 6.95 \\
\hline & RES & $2.93 e-05$ & $7.31 \mathrm{e}-04$ \\
\hline & ERR & $1.65 \mathrm{e}-03$ & $7.08 \mathrm{e}-03$ \\
\hline \multirow[t]{4}{*}{$10^{-4}$} & Iter & $8(81)$ & $8(203)$ \\
\hline & $\mathrm{CPU}$ & 1.25 & 6.74 \\
\hline & RES & $3.93 e-06$ & $4.02 \mathrm{e}-06$ \\
\hline & ERR & $3.41 \mathrm{e}-05$ & $3.50 \mathrm{e}-05$ \\
\hline \multirow[t]{4}{*}{$10^{-3}$} & Iter & $14(47)$ & $14(49)$ \\
\hline & $\mathrm{CPU}$ & 1.30 & 4.59 \\
\hline & RES & $1.17 \mathrm{e}-07$ & $1.17 \mathrm{e}-07$ \\
\hline & ERR & $1.79 \mathrm{e}-06$ & $1.80 \mathrm{e}-06$ \\
\hline \multirow[t]{4}{*}{$10^{-2}$} & Iter & $27(23)$ & $27(24)$ \\
\hline & $\mathrm{CPU}$ & 1.44 & 3.37 \\
\hline & RES & $1.18 \mathrm{e}-08$ & $3.93 e-08$ \\
\hline & ERR & $1.69 \mathrm{e}-07$ & $4.26 \mathrm{e}-07$ \\
\hline \multirow[t]{4}{*}{$10^{-1}$} & Iter & $40(17)$ & $40(18)$ \\
\hline & $\mathrm{CPU}$ & 1.54 & 4.05 \\
\hline & RES & $5.43 e-08$ & $3.85 \mathrm{e}-08$ \\
\hline & ERR & $1.18 \mathrm{e}-06$ & $7.50 \mathrm{e}-07$ \\
\hline \multirow[t]{4}{*}{1} & Iter & $42(16)$ & $42(17)$ \\
\hline & $\mathrm{CPU}$ & 1.66 & 3.48 \\
\hline & RES & $1.10 \mathrm{e}-08$ & $2.01 \mathrm{e}-08$ \\
\hline & ERR & $3.37 \mathrm{e}-07$ & $2.82 \mathrm{e}-07$ \\
\hline
\end{tabular}

subspace methods for multiple linear systems, J. Comput. Appl. Math., 196 (2006), pp. 498-511.

[10] G. EBADI, N. AliPOUR, AND C. VUIK, Deflated and augmented global Krylov subspace methods for the matrix equations, Appl. Numer. Math., 99 (2016), pp. 137-150.

[11] A. El Guennouni, K. JBILOU, And H. SADOK, A Block version of BICGSTAB for Linear systems with multiple right-hand sides, Electron. Trans. Numer. Anal., 16 (2003), pp. 129-142.

http://etna.ricam.oeaw.ac.at/vol.16.2003/pp129-142.dir/pp129-142.pdf

[12] H. C. Elman, D. J. Silvester, And A. J. Wathen, Finite Elements and Fast Iterative Solvers with Applications in Incompressible Fluid Dynamics, Oxford University Press, New York, 2005.

[13] Z.-G. HuAnG, G.-L. WANG, Z. XU, AND J.-J. CUI, A generalized variant of the deteriorated PSS preconditioner for nonsymmetric saddle point problems, Numer. Algorithms, 75 (2017), pp. 1161-1191.

[14] K. JBILOU, A. MESSAOUdi, AND H. SADOK, Global FOM and GMRES algorithms for matrix equation, Appl. Numer. Math., 31 (1999), pp. 49-43.

[15] K. JBILOU, H. SAdoK, AND A. TinzeFTE, Oblique projection methods for linear systems with multiple right-hand sides, Electron. Trans. Numer. Anal., 20 (2005), pp. 119-138.

http: / / etna.ricam. oeaw.ac.at/vol.20.2005/pp119-138.dir/pp119-138.pdf

[16] Y. SAAD, A flexible inner-outer preconditioned GMRES algorithm, SIAM J. Sci. Comput., 14 (1993), pp. 461469. 